\title{
Pion pressure in a magnetic field
}

\author{
Christoph P. Hofmann \\ Facultad de Ciencias, Universidad de Colima, Bernal Díaz del Castillo 340, Colima C.P. 28045, Mexico
}

(Received 20 April 2020; accepted 11 June 2020; published 29 June 2020)

\begin{abstract}
While the partition function for QCD in a magnetic field $H$ has been calculated before within chiral perturbation theory up to two-loop order, our investigation relies on an alternative representation for the Bose functions which allows for a clear-cut expansion of thermodynamic quantities in the chiral limit. We first focus on the pion-pion interaction in the pressure and show that-depending on magnetic field strength, temperature, and pion mass - it may be attractive or repulsive. We then analyze the thermodynamic properties in the chiral limit and provide explicit two-loop representations for the pressure in the weak magnetic field limit $|q H| \ll T^{2}$.
\end{abstract}

DOI: 10.1103/PhysRevD.101.114031

\section{INTRODUCTION}

The low-energy properties of quantum chromodynamics (QCD) can be understood on the basis of its relevant lowenergy degrees of freedom: the Goldstone bosons. This is the path pursued by chiral perturbation theory (CHPT), and indeed, the low-temperature properties of $\mathrm{QCD}$ in a magnetic field have been explored within CHPT in many studies up to two-loop order [1-12]. Other approaches to finite-temperature QCD in magnetic fields include lattice QCD [13-25], Nambu-Jona-Lasinio model-based studies [26-33], and other techniques [34-60]. Yet more references can be found in the review of Ref. [61].

Recently, the present author has pointed out that the chiral expansion of the quark condensate in a weak magnetic field and in the chiral limit has not been properly derived, and has provided the correct series in Ref. [62]. ${ }^{1}$ The analysis was based on an alternative representation for the Bose functions, which was the key to deriving the proper series in a transparent manner. Relying on this coordinate space representation, here we take the analysis up to the two-loop level. This does not merely correspond to rederiving or rephrasing known results for QCD in

\footnotetext{
${ }^{1}$ The error in Refs. [2-4,8] appears to be related to an incorrect expansion of the function $\varphi$ in the limit of weak magnetic fields. The mistake in Refs. $[9,10]$ can be traced back to the EulerMcLaurin expansion of thermodynamic functions, where not all relevant terms have been taken into account. A more detailed analysis of the series given in Refs. [9,10], as well as a discussion of the discrepancy with respect to my series in Ref. [62], is forthcoming [63].

Published by the American Physical Society under the terms of the Creative Commons Attribution 4.0 International license. Further distribution of this work must maintain attribution to the author(s) and the published article's title, journal citation, and DOI. Funded by SCOAP ${ }^{3}$.
}

magnetic fields in an alternative framework. Rather, our analysis goes beyond the literature by (i) analyzing how the nature of the pion-pion interaction in the pressurerepulsive or attractive-is affected by the magnetic field, as well as the temperature and pion mass, and (ii) providing the chiral expansion for the pressure in weak magnetic fields $\left(|q H| \ll T^{2}\right)$ in the chiral limit.

As different expansions and limits are considered in the present work, it is useful to briefly make some pertinent comments. First of all, the chiral expansion on which CHPT relies is an expansion valid at low momentum, energy, or temperature. The chiral expansion is organized according to the number of loops, and in three spatial dimensions each loop is suppressed by two momentum (temperature) powers. In this sense, we are dealing with a low-temperature expansion, because temperature-as well as the pion masses and magnetic field-has to be small compared to the chiral-symmetry-breaking scale $\Lambda_{\chi}$, which is approximately $1 \mathrm{GeV}$. While $T, M_{\pi}$, and $H$ must be small, the ratios between them can take any values. In the present work, we are interested in the chiral limit that is achieved by taking the limit $M_{\pi} \rightarrow 0$ at fixed temperature: the ratio $M_{\pi} / T$ tends to zero, which corresponds to addressing the regime $M_{\pi} \ll T$. Therefore, although temperature is still low compared to $\Lambda_{\chi}$, we are dealing here with a high-temperature expansion or high-temperature limit. Once the chiral limit at fixed temperature has been taken, we then consider another limit, namely $|q H| \ll T^{2}$. Again, taking the limit $|q H| \rightarrow 0$ at fixed temperature corresponds to a high-temperature expansion. Throughout the paper, we will refer to the limit $|q H| \ll T^{2}$ as the weak magnetic field limit.

In terms of the dressed pions at zero temperature, the chiral expansion of the pressure in a magnetic field takes a remarkably simple form. Noninteracting pions yield the well-known $T^{4}$ contribution, while interaction effects enter 
at order $T^{6}$. In the chiral limit $(M \rightarrow 0)$-irrespective of whether or not the magnetic field is present-the two-loop interaction contribution vanishes. In the case $M \neq 0$, the pion-pion interaction in the pressure is mostly attractive, but it may become repulsive at low temperatures as the magnetic field strength grows. In general, the sign and magnitude of the interaction depend on the actual values of temperature, magnetic field, and pion masses in a nontrivial way, as we illustrate in various figures.

In the chiral limit, the expansion of the pressure in a weak magnetic field $H$ is dominated by terms involving $\epsilon^{3 / 2}, \epsilon^{2} \log \epsilon$, and $\epsilon^{2}$, where $\epsilon=|q H| / T^{2}$ is the relevant expansion parameter and $q$ is the electric charge of the pion.

The article is organized as follows: The evaluation of the QCD partition function in a magnetic field up to twoloop order within chiral perturbation theory is presented in Sec. II. The nature of the pion-pion interaction in the pressure-attractive or repulsive-is analyzed in Sec. III. We then focus in Sec. IV on the thermodynamic properties in the chiral limit and provide the weak magnetic field expansion of the pressure to arbitrary order in $\epsilon=|q H| / T^{2}$. Finally, in Sec. V we conclude. While details on the two-loop CHPT evaluation are discussed in Appendix A, the rather technical analysis of the pressure in weak magnetic fields in the chiral limit is presented in Appendix B.

\section{CHIRAL PERTURBATION THEORY EVALUATION}

\section{A. Preliminaries}

Surveys of chiral perturbation theory have been provided on many occasions (see, e.g., Refs. [64,65]) - in what follows, we only touch upon the very basic elements to set the notation. Throughout the study, we refer to the isospin limit $m_{u}=m_{d}$. $U(x)$,

The effective pion fields $\pi^{i}(x)$ appear in the $\mathrm{SU}(2)$ matrix

$$
U(x)=\exp \left(i \tau^{i} \pi^{i}(x) / F\right), \quad i=1,2,3,
$$

where $\tau^{i}$ are Pauli matrices and $F$ represents the pion decay constant at tree level. The leading piece in the effective Lagrangian is of momentum order $p^{2}$ and takes the form

$$
\mathcal{L}_{\text {eff }}^{2}=\frac{1}{4} F^{2} \operatorname{Tr}\left[\left(D_{\mu} U\right)^{\dagger}\left(D_{\mu} U\right)-M^{2}\left(U+U^{\dagger}\right)\right] .
$$

Here $M$ is the pion mass at tree level. The magnetic field $H$ enters via the covariant derivative

$$
D_{\mu} U=\partial_{\mu} U+i[Q, U] A_{\mu}^{\mathrm{EM}},
$$

where $Q$ is the charge matrix of the quarks, $Q=\operatorname{diag}(2 / 3$, $-1 / 3) e$. The gauge field $A_{\mu}^{\mathrm{EM}}=\left(0,0,-H x_{1}, 0\right)$ contains the (constant) magnetic field [61].

In the present analysis, we also need higher-order pieces of the effective Lagrangian, namely $\mathcal{L}_{\text {eff }}^{4}$ and $\mathcal{L}_{\text {eff }}^{6}$. The explicit structure is given, e.g., in Refs. [66,67]. The relevant Feynman diagrams for the partition function up to two-loop order $p^{6}$ are shown in Fig. 1. The lines represent thermal propagators which correspond either to the charged pions or to the neutral pion. In fact, the dimensionally regularized zero-temperature propagator $\Delta^{0}(x)$ for the neutral pion in Euclidean space takes the familiar form

$$
\begin{aligned}
\Delta^{0}(x) & =(2 \pi)^{-d} \int \mathrm{d}^{d} p e^{i p x}\left(M^{2}+p^{2}\right)^{-1} \\
& =\int_{0}^{\infty} \mathrm{d} \rho(4 \pi \rho)^{-d / 2} e^{-\rho M^{2}-x^{2} / 4 \rho} .
\end{aligned}
$$

On the other hand, the dimensionally regularized zerotemperature propagator $\Delta^{ \pm}(x)$ for the charged pions does involve the magnetic field. In Euclidean space, as derived in Ref. [62], it amounts to

$$
\begin{aligned}
\Delta^{ \pm}(x)= & \frac{|q H|}{(4 \pi)^{\frac{d}{2}}} e^{-s_{\perp}|q H| x_{1} x_{2} / 2} \int_{0}^{\infty} \mathrm{d} \rho \frac{\rho^{-\frac{d}{2}+1} e^{-\rho M^{2}}}{\sinh (|q H| \rho)} \\
& \times \exp \left[-\frac{x_{4}^{2}+x_{3}^{2}}{4 \rho}-\frac{|q H|\left(x_{1}^{2}+x_{2}^{2}\right)}{4 \tanh (|q H| \rho)}\right],
\end{aligned}
$$

where

$$
s_{\perp}=\operatorname{sign}(q H) .
$$

In either case-for neutral and charged pions-the thermal propagators are obtained by summing over zerotemperature propagators as

$$
G(x)=\sum_{n=-\infty}^{\infty} \Delta\left(\vec{x}, x_{4}+n \beta\right), \quad \beta=\frac{1}{T} .
$$

In the evaluation of the partition function diagrams displayed in Fig. 1, as we will see, thermal propagators only have to be considered at the origin $x=0$. It is furthermore advantageous to isolate the zero-temperature pieces $\Delta^{ \pm}$and $\Delta^{0}$ in the thermal propagators via

$$
\begin{aligned}
G^{ \pm}(0) & \equiv G_{1}^{ \pm}=\Delta^{ \pm}(0)+g_{1}^{ \pm}(M, T, H), \\
G^{0}(0) & \equiv G_{1}^{0}=\Delta^{0}(0)+g_{1}(M, T, 0) .
\end{aligned}
$$

The quantities $g_{1}^{ \pm}(M, T, H)$ and $g_{1}(M, T, 0)$ are kinematical functions that describe the purely finite-temperature part. They are embedded in the more general class of Bose functions defined by 


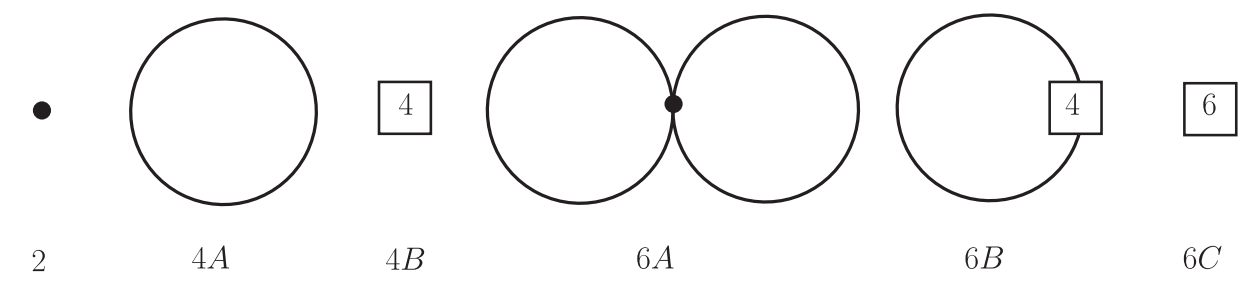

FIG. 1. QCD partition function diagrams up to order $p^{6} \propto T^{6}$. The filled circle stands for $\mathcal{L}_{\text {eff }}^{2}$; the numbers 4 and 6 in the boxes represent $\mathcal{L}_{\text {eff }}^{4}$ and $\mathcal{L}_{\text {eff }}^{6}$.

$$
\begin{aligned}
g_{r}^{ \pm}(M, T, H) & =\frac{T^{d-2 r-2}}{(4 \pi)^{r+1}}|q H| \int_{0}^{\infty} \mathrm{d} \rho \frac{\rho^{r-\frac{d}{2}}}{\sinh \left(|q H| \rho / 4 \pi T^{2}\right)} \exp \left(-\frac{M^{2}}{4 \pi T^{2}} \rho\right)\left[S\left(\frac{1}{\rho}\right)-1\right], \\
g_{r}(M, T, 0) & =\frac{T^{d-2 r}}{(4 \pi)^{r}} \int_{0}^{\infty} \mathrm{d} \rho \rho^{r-\frac{d}{2}-1} \exp \left(-\frac{M^{2}}{4 \pi T^{2}} \rho\right)\left[S\left(\frac{1}{\rho}\right)-1\right], \\
S(z) & =\sum_{n=-\infty}^{\infty} \exp \left(-\pi n^{2} z\right),
\end{aligned}
$$

where $S(z)$ is the Jacobi theta function. Note that $g_{r}(M, T, 0)$ does not involve the magnetic field. In order to facilitate the subsequent analysis, in the Bose functions $g_{r}^{ \pm}(M, T, H)$ for the charged pions, we extract the $H=0$ part as

$$
g_{r}^{ \pm}(M, T, H)=\tilde{g}_{r}(M, T, H)+g_{r}(M, T, 0),
$$

where solely

$$
\tilde{g}_{r}(M, T, H)=\frac{T^{d-2 r-2}}{(4 \pi)^{r+1}}|q H| \int_{0}^{\infty} \mathrm{d} \rho \rho^{r-\frac{d}{2}}\left(\frac{1}{\sinh \left(|q H| \rho / 4 \pi T^{2}\right)}-\frac{4 \pi T^{2}}{|q H| \rho}\right) \times \exp \left(-\frac{M^{2}}{4 \pi T^{2}} \rho\right)\left[S\left(\frac{1}{\rho}\right)-1\right]
$$

contains the magnetic field. These two types of kinematical functions- $\tilde{g}_{r}(M, T, H)$ and $g_{r}(M, T, 0)$-constitute the basic building blocks in our analysis. The decomposition of the thermal propagators into $T=0$ and finite- $T$ pieces then results in

$$
\begin{aligned}
G_{1}^{ \pm} & =\Delta^{ \pm}(0)+\tilde{g}_{1}(M, T, H)+g_{1}(M, T, 0), \\
G_{1}^{0} & =\Delta^{0}(0)+g_{1}(M, T, 0) .
\end{aligned}
$$

As a low-energy effective field theory, chiral perturbation theory describes QCD in the regime where quark masses as small, magnetic fields are weak, and temperatures are low: the quantities $M, H$, and $T$ ought to be small compared to the chiral-symmetry-breaking scale $\Lambda_{\chi} \approx 1 \mathrm{GeV}$. While ratios between these parameters in principle can have any value, in the present analysis, the limits $M / T \rightarrow 0$ (chiral limit at fixed temperature) and $|q H| \ll T^{2}$ (weak magnetic field limit) are of particular interest.

\section{B. Free energy density up to order $p^{6}$}

The one-loop free energy density (order $p^{4}$ )—in coordinate space representation-has been derived in Ref. [62]. The final renormalized expression reads

$$
z_{2+4 A+4 B}=z_{0}^{[4]}-\frac{3}{2} g_{0}(M, T, 0)-\tilde{g}_{0}(M, T, H) .
$$

The zero-temperature part $z_{0}^{[4]}$ is ${ }^{2}$

$$
\begin{aligned}
z_{0}^{[4]}= & -F^{2} M^{2}+\frac{M^{4}}{64 \pi^{2}}\left(\bar{l}_{3}-4 \bar{h}_{1}-\frac{3}{2}\right)+\frac{|q H|^{2}}{96 \pi^{2}}\left(\bar{h}_{2}-1\right) \\
& -\frac{|q H|^{2}}{16 \pi^{2}} \int_{0}^{\infty} \mathrm{d} \rho \rho^{-2}\left(\frac{1}{\sinh (\rho)}-\frac{1}{\rho}+\frac{\rho}{6}\right) \\
& \times \exp \left(-\frac{M^{2}}{|q H|} \rho\right)
\end{aligned}
$$

Modulo factors of $\gamma_{3} / 32 \pi^{2}, \delta_{1} / 32 \pi^{2}$, and $\delta_{2} / 32 \pi^{2}$, the quantities $\bar{l}_{3}, \bar{h}_{1}$, and $\bar{h}_{2}$ represent the running effective coupling constants evaluated at the renormalization scale $\mu=M_{\pi}\left(M_{\pi} \approx 140 \mathrm{MeV}\right)$-details can be found, e.g., in Ref. [68].

At the two-loop level (order $p^{6}$ ), the three partition function diagrams $6 A-6 C$ have to be evaluated-this is done in Appendix A. In terms of the tree-level pion mass $M$, the outcome is

\footnotetext{
${ }^{2}$ The third term in the first parenthesis should read $-\frac{3}{2}$. In Ref. [62], Eq. (A7), it was inadvertently cited as -1 .
} 


$$
\begin{aligned}
z_{6 A+6 B+6 C}= & z_{0}^{[6]}+\frac{3 M^{2}}{8 F^{2}}\left(g_{1}\right)^{2}+\frac{M^{2}}{2 F^{2}} g_{1} \tilde{g}_{1} \\
& +g_{1}\left[-\frac{3 \bar{l}_{3}}{64 \pi^{2}} \frac{M^{4}}{F^{2}}+\frac{M^{2}}{2 F^{2}} K_{1}+\frac{\bar{l}_{6}-\bar{l}_{5}}{48 \pi^{2}} \frac{|q H|^{2}}{F^{2}}\right] \\
& +\tilde{g}_{1}\left[-\frac{\bar{l}_{3}}{32 \pi^{2}} \frac{M^{4}}{F^{2}}+\frac{\bar{l}_{6}-\bar{l}_{5}}{48 \pi^{2}} \frac{|q H|^{2}}{F^{2}}\right]
\end{aligned}
$$

where the integral $K_{1}$ is defined in Eq. (A5). Since we are interested in the behavior of the system at finite temperature, the explicit structure of the $T=0$ contribution $z_{0}^{[6]}$ is not needed here.

Let us have a closer look at the terms linear in $g_{1}$ and $\tilde{g}_{1}$. First, notice that the kinematical functions $g_{1}$ and $\tilde{g}_{1}$ are related to $g_{0}$ and $\tilde{g}_{0}$ through

$$
g_{1}=-\frac{\partial g_{0}}{\partial M^{2}}, \quad \tilde{g}_{1}=-\frac{\partial \tilde{g}_{0}}{\partial M^{2}} .
$$

In the presence of a magnetic field, the mass of a charged pion $\left(M_{\pi}^{ \pm}\right)$is different from the mass of a neutral pion $\left(M_{\pi}^{0}\right)$. In order to determine these masses, we express the kinematical functions $g_{0}$ and $\tilde{g}_{0}$ in terms of $M_{\pi}^{ \pm}$and $M_{\pi}^{0}$ instead of $M$. Since only the charged pions are tied to $\tilde{g}_{r}{ }^{3}$ we can write

$$
\tilde{g}_{0}\left(M_{\pi}^{ \pm}, T, H\right)=\tilde{g}_{0}(M, T, H)-\tilde{g}_{1}(M, T, H) \tilde{\epsilon}_{1},
$$

where $\tilde{\epsilon}_{1}$ measures the mass-squared difference

$$
\tilde{\epsilon}_{1}=\left(M_{\pi}^{ \pm}\right)^{2}-M^{2}
$$

Comparing with the third line of Eq. (2.15), we identify $\tilde{\epsilon}_{1}$ as

$$
\tilde{\epsilon}_{1}=\frac{\bar{l}_{6}-\bar{l}_{5}}{48 \pi^{2}} \frac{|q H|^{2}}{F^{2}}-\frac{\bar{l}_{3}}{32 \pi^{2}} \frac{M^{4}}{F^{2}} .
$$

As for $g_{r}$-where all three pions are involved, according to Eq. (2.12)—we must distinguish between the respective pieces: for the charged pions we write

$$
g_{0}\left(M_{\pi}^{ \pm}, T, 0\right)=g_{0}(M, T, 0)-g_{1}(M, T, 0) \tilde{\epsilon}_{1},
$$

while for the neutral pion we have

$$
g_{0}\left(M_{\pi}^{0}, T, 0\right)=g_{0}(M, T, 0)-g_{1}(M, T, 0) \epsilon_{1} .
$$

The quantity $\epsilon_{1}$ measures the mass-squared difference

$$
\epsilon_{1}=\left(M_{\pi}^{0}\right)^{2}-M^{2}
$$

and can be identified as

\footnotetext{
${ }^{3}$ See Eq. (2.12).
}

$$
\epsilon_{1}=-\frac{\bar{l}_{3}}{32 \pi^{2}} \frac{M^{4}}{F^{2}}+\frac{M^{2}}{F^{2}} K_{1} .
$$

As a result, we can read off how the pion masses are affected by the magnetic field:

$$
\begin{aligned}
& \left(M_{\pi}^{ \pm}\right)^{2}=M_{\pi}^{2}+\frac{\bar{l}_{6}-\bar{l}_{5}}{48 \pi^{2}} \frac{|q H|^{2}}{F^{2}}, \\
& \left(M_{\pi}^{0}\right)^{2}=M_{\pi}^{2}+\frac{M^{2}}{F^{2}} K_{1} .
\end{aligned}
$$

Note that $M_{\pi}$ is the pion mass in zero magnetic field, given by

$$
M_{\pi}^{2}=M^{2}-\frac{\bar{l}_{3}}{32 \pi^{2}} \frac{M^{4}}{F^{2}}+\mathcal{O}\left(M^{6}\right) .
$$

The mass relations in Eq. (2.24) indeed coincide with those obtained by Andersen in Ref. [10]—-see Eqs. (3.8)(3.10) — in the zero-temperature limit. It should be pointed out that we consider the pion masses at zero temperature, while in Ref. [10] finite-temperature effects are included as well. As it turns out, to have a clear definition of interaction effects in the thermodynamic quantities, we must consider the pion masses at $T=0$ i.e., dress the pions at zero temperature according to Eq. (2.24).

The result for the total two-loop free energy density simplifies considerably if we now express the kinematical functions $g_{0}$ and $\tilde{g}_{0}$ in the one-loop contributionEq. (2.13) — by the masses $M_{\pi}^{ \pm}$and $M_{\pi}^{0}$ rather than by $M$. Using Eqs. (2.17), (2.20), and (2.21), we obtain

$$
\begin{aligned}
z_{\mathrm{tot}}= & z_{0}-g_{0}\left(M_{\pi}^{ \pm}, T, 0\right)-\frac{1}{2} g_{0}\left(M_{\pi}^{0}, T, 0\right)-\tilde{g}_{0}\left(M_{\pi}^{ \pm}, T, H\right) \\
& +\frac{M_{\pi}^{2}}{2 F^{2}} g_{1}\left(M_{\pi}^{ \pm}, T, 0\right) g_{1}\left(M_{\pi}^{0}, T, 0\right) \\
& -\frac{M_{\pi}^{2}}{8 F^{2}}\left\{g_{1}\left(M_{\pi}^{0}, T, 0\right)\right\}^{2} \\
& +\frac{M_{\pi}^{2}}{2 F^{2}} g_{1}\left(M_{\pi}^{0}, T, 0\right) \tilde{g}_{1}\left(M_{\pi}^{ \pm}, T, H\right)+\mathcal{O}\left(p^{8}\right),
\end{aligned}
$$

where $z_{0}$ is the zero-temperature piece. The crucial point is that all terms linear in $g_{1}(M, T, 0)$ and $\tilde{g}_{1}(M, T, H)$ have been absorbed into mass renormalization: $M^{2} \rightarrow$ $\left(M_{\pi}^{ \pm}\right)^{2},\left(M_{\pi}^{0}\right)^{2}$. In particular, the effect of the pion-pion interaction at finite temperature is solely contained in the terms quadratic in the kinematical functions. It should be noted that the differences between $M_{\pi}^{2}$, Eq. (2.25), and the tree-level mass $M^{2}$ at the order we are considering are irrelevant in the coefficients accompanying the terms quadratic in the kinematical functions, such that it is legitimate to write $M_{\pi}^{2}$. 
While the evaluation of the two-loop free energy density in Refs. $[9,10]$ is based on a momentum-space representation for the kinematical functions, here we have used an alternative representation based on coordinate space. The advantage is that the latter approach allows for a clear-cut expansion of thermodynamic quantities in the chiral limit, as we demonstrate below.

\section{PRESSURE: NATURE OF PION-PION INTERACTION}

We now explore the manifestation of the pion-pion interaction in the pressure, which we derive from the two-loop free energy density as

$$
P=z_{0}-z_{\text {tot }} .
$$
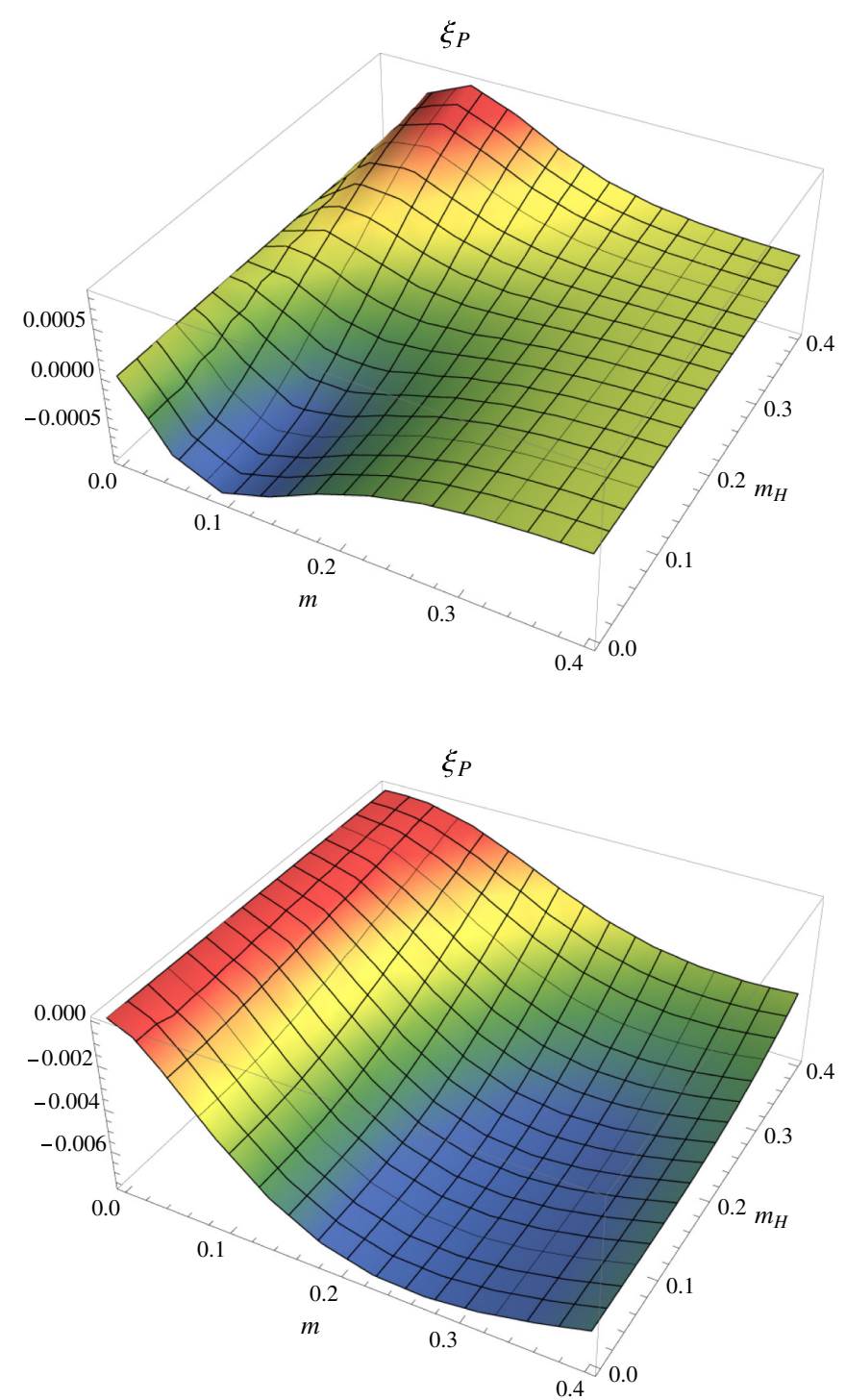

To make temperature powers in the pressure manifest, we replace the Bose functions $g_{r}$ and $\tilde{g}_{r}$ with the dimensionless kinematical functions $h_{r}$ and $\tilde{h}_{r}$ according to

$$
h_{0}=\frac{g_{0}}{T^{4}}, \quad \tilde{h}_{0}=\frac{\tilde{g}_{0}}{T^{4}}, \quad h_{1}=\frac{g_{1}}{T^{2}}, \quad \tilde{h}_{1}=\frac{\tilde{g}_{1}}{T^{2}}
$$

and obtain the low-temperature expansion of the pressure as

$P=p_{1}\left(t, m, m_{H}\right) T^{4}+p_{2}\left(t, m, m_{H}\right) T^{6}+\mathcal{O}\left(T^{8}\right)$,

with the coefficients
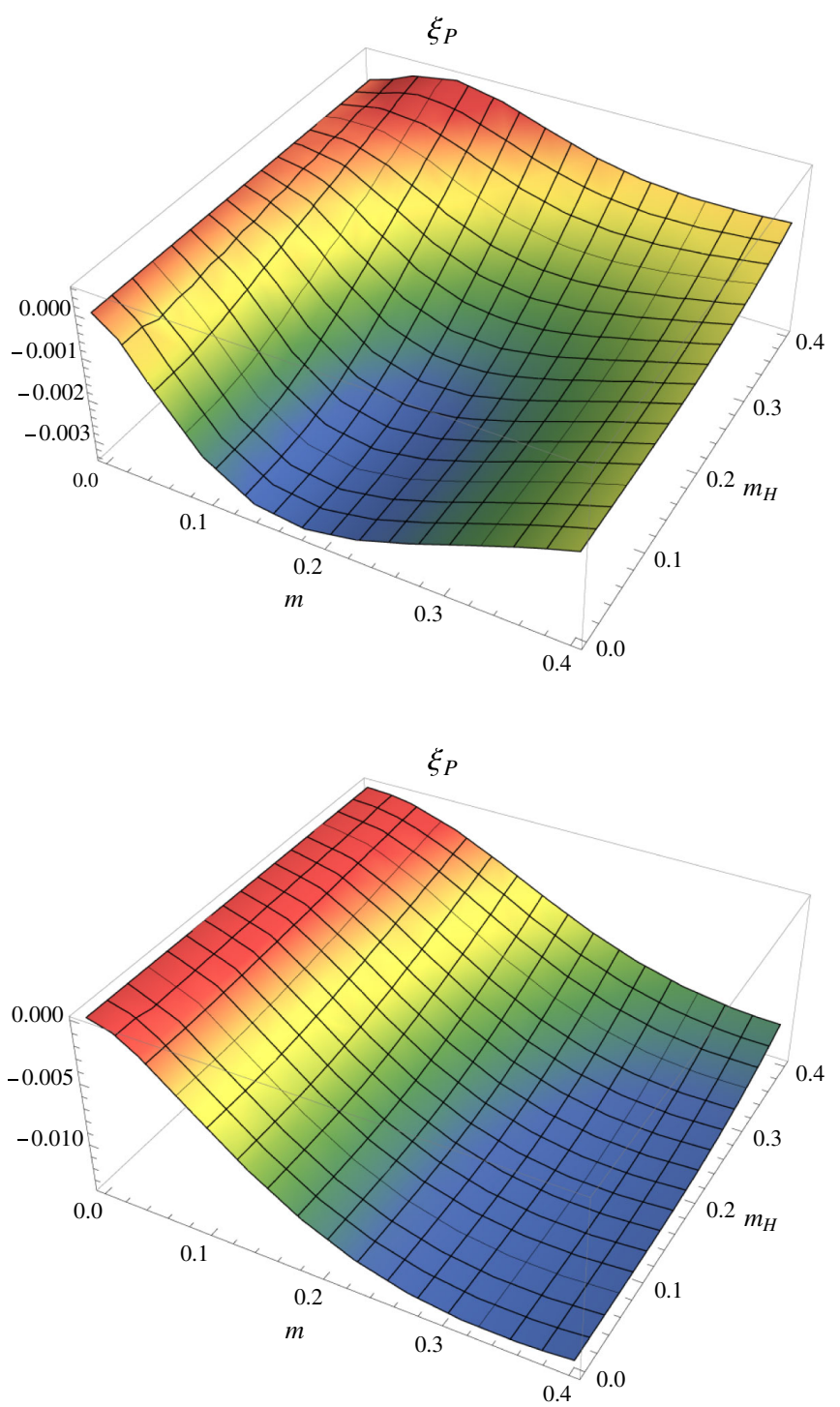

FIG. 2. Magnitude and sign of the pion-pion interaction in the pressure measured by $\xi_{P}\left(t, m, m_{H}\right)$-Eq. (3.6)—for the temperatures $T=53.8 \mathrm{MeV}$ and $108 \mathrm{MeV}$ (upper panels), and $T=161 \mathrm{MeV}$ and $215 \mathrm{MeV}$ (lower panels). 


$$
\begin{aligned}
p_{1}\left(t, m, m_{H}\right)= & h_{0}\left(M_{\pi}^{ \pm}, T, 0\right)+\frac{1}{2} h_{0}\left(M_{\pi}^{0}, T, 0\right) \\
& +\tilde{h}_{0}\left(M_{\pi}^{ \pm}, T, H\right), \\
p_{2}\left(t, m, m_{H}\right)= & -\frac{m^{2}}{2 t^{2} F^{2}} h_{1}\left(M_{\pi}^{ \pm}, T, 0\right) h_{1}\left(M_{\pi}^{0}, T, 0\right) \\
& +\frac{m^{2}}{8 t^{2} F^{2}}\left\{h_{1}\left(M_{\pi}^{0}, T, 0\right)\right\}^{2} \\
& -\frac{m^{2}}{2 t^{2} F^{2}} h_{1}\left(M_{\pi}^{0}, T, 0\right) \tilde{h}_{1}\left(M_{\pi}^{ \pm}, T, H\right) .
\end{aligned}
$$

The dimensionless parameters $t, m$, and $m_{H}$,

$$
t=\frac{T}{4 \pi F}, \quad m=\frac{M_{\pi}}{4 \pi F}, \quad m_{H}=\frac{\sqrt{|q H|}}{4 \pi F},
$$

measure the temperature, pion mass $M_{\pi}$ [Eq. (2.25)], and magnetic field strength with respect to the scale $4 \pi F \approx \Lambda_{\chi}$-i.e., with respect to the chiral-symmetrybreaking scale $\Lambda_{\chi} \approx 1 \mathrm{GeV}$. In the domain where chiral perturbation theory operates, these parameters are small: more concretely, in the plots below, we will restrict ourselves to the parameter region $t, m, m_{H} \lesssim 0.3$. For the pion masses we use $M_{\pi}=140 \mathrm{MeV}$ and, following Ref. [69], for the pion decay constant we get $F=85.6 \mathrm{MeV}$. Finally, according to Ref. [66], for the combination of NLO low-energy constants as it appears in the charged pion masses, we take $\bar{l}_{6}-\bar{l}_{5}=2.64$.

The $T^{4}$ contribution in the low-temperature series for the pressure corresponds to the noninteracting pion gas, while the pion-pion interaction emerges at order $T^{6}$. Recall that the Bose functions $h_{0}$ and $h_{1}$ do not explicitly involve the magnetic field: the effect of the magnetic field is embedded in the Bose functions $\tilde{h}_{0}$ and $\tilde{h}_{1}$. In the chiral limit $(M \rightarrow 0)$, the coefficient $p_{2}\left(t, m, m_{H}\right)$ tends to zero: the pion-pion interaction only starts manifesting itself at the three-loop level, as is well known for the case $H=0$ (see, e.g., Ref. [68]). However, for $M \neq 0$, the interaction term is present, and-depending on the actual values of the parameters $t, m$, and $m_{H}$ - the resulting pion-pion interaction in the pressure may be attractive or repulsive, as we now illustrate.

To get a more quantitative picture, let us consider the dimensionless ratio

$$
\xi_{P}\left(t, m, m_{H}\right)=\frac{p_{2}\left(t, m, m_{H}\right) T^{2}}{p_{1}\left(t, m, m_{H}\right)}
$$

that measures the magnitude and sign of the pionpion interaction relative to the noninteracting pion gas contribution. In Fig. 2, we depict this ratio for the four temperatures $t=\{0.05,0.1,0.15,0.2\}$, or equivalently, $T=\{53.8,108,161,215\} \mathrm{MeV}$.

In the limit $M \rightarrow 0$, irrespective of absence or presence of the magnetic field, the two-loop interaction contribution vanishes. In the other limit, $H \rightarrow 0$, the interaction in the

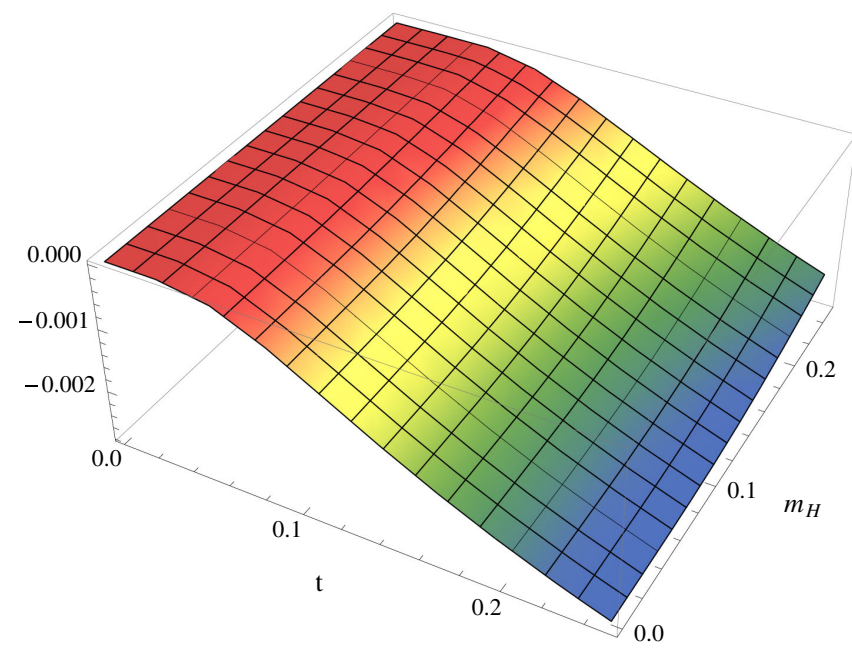

FIG. 3. Magnitude and sign of the pion-pion interaction in the QCD pressure as a function of temperature $(t)$ and magnetic field strength $\left(m_{H}\right)$-measured by $p_{2}\left(t, m, m_{H}\right) T^{2}$-at the physical value $M_{\pi}=140 \mathrm{MeV}$ of the pion masses.

pressure always is attractive, irrespective of the actual values of the (nonzero) pion masses and temperature. When the magnetic field is switched on, the attractive pion-pion interaction becomes weaker, but only at low temperatures and stronger magnetic fields does the pionpion interaction become repulsive. Overall, the interaction in the pressure is quite small, at most around 1\% compared to the leading free Bose gas contribution. It remains to be seen whether these subtle effects in the interaction can be observed in numerical lattice QCD simulations-in particular, the repulsive effect at lower temperatures and smaller pion masses.

The case of interest ${ }^{4}$ corresponding to the physical value of the pion masses- $M_{\pi}=140 \mathrm{MeV}$, i.e., $m=0.130$-is depicted in Fig. 3, where we plot the dimensionless two-loop contribution $p_{2}\left(t, m, m_{H}\right) T^{2}$ as a function of temperature and magnetic field strength. As the figure suggests, the interaction is purely attractive in the parameter domain $t, m_{H} \leq 0.25$. As the strength of the magnetic field grows, the attractive interaction gradually becomes weaker. Note that the maximal values for the parameters $t$ and $m_{H}$ correspond to $T \approx 269 \mathrm{MeV}$ and $\sqrt{|q H|} \approx 269 \mathrm{MeV}$, respectively. In other words, we are already in a region where temperature and magnetic field strength are no longer small compared to the underlying scale $\Lambda_{\chi}$ and the low-temperature expansion starts to break down.

\section{PRESSURE IN WEAK MAGNETIC FIELDS IN THE CHIRAL LIMIT}

The objective of Ref. [62] was to provide the correct series for the one-loop quark condensate in weak magnetic

\footnotetext{
${ }^{4}$ Note that we refer to the isospin limit where all three pions have the same mass (in the absence of the magnetic field).
} 


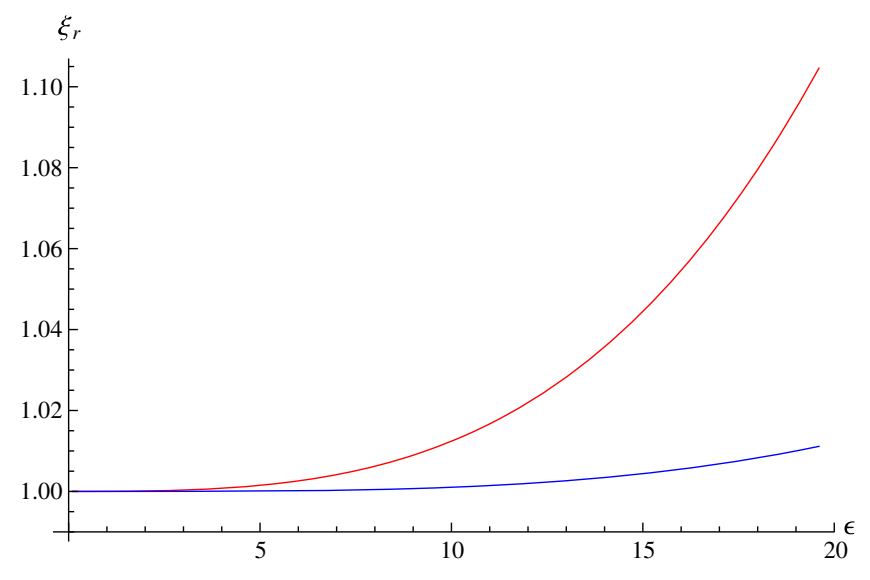

FIG. 4. Comparison of the first three terms in the series for the functions $\tilde{g}_{0}$ (red curve) and $\tilde{g}_{1}$ (blue curve) with respect to the full functions.

fields in the chiral limit. The corresponding analysis involved the kinematical function $\tilde{g}_{1}$. Regarding the pressure, the one-loop contribution involves the kinematical function $\tilde{g}_{0}$. In Appendix B, we derive the expansion of this function in weak magnetic fields in the chiral limit, following analogous strategies as for $\tilde{g}_{1}$. Based on these results, we now discuss the structure of the weak magnetic field expansion of the pressure in the chiral limit up to twoloop order, which is new to the best of our knowledge.

In the chiral limit, as stated previously, the pion-pion interaction in the pressure starts showing up only at threeloop order, which is beyond the scope of our investigation. The series for the pressure in the chiral limit is hence fixed by the Bose contribution of order $T^{4}$ that contains the kinematical function $\tilde{g}_{0}$. With the weak magnetic field expansion for $\tilde{g}_{0}$, Eq. (B27), the series for the pressure in weak magnetic fields and in the chiral limit takes the form

$$
\begin{aligned}
P= & \frac{\pi^{2}}{30} T^{4}+\left\{-\frac{\left|I_{\frac{3}{2}}\right|}{8 \pi^{3 / 2}} \epsilon^{\frac{3}{2}}-\frac{1}{96 \pi^{2}} \epsilon^{2} \log \epsilon+b_{1} \epsilon^{2}+\mathcal{O}\left(\epsilon^{4}\right)\right\} T^{4} \\
& -\left(\bar{l}_{6}-\bar{l}_{5}\right)\left\{\frac{t^{2}}{36} \epsilon^{2}-\frac{t^{2}\left|I_{\frac{1}{2}}\right|}{24 \pi^{3 / 2}} \epsilon^{\frac{5}{2}}+\frac{t^{2} \log 2}{48 \pi^{2}} \epsilon^{3}+\mathcal{O}\left(\epsilon^{\frac{7}{2}}\right)\right\} T^{4} \\
& +\mathcal{O}\left(T^{8} \log T\right),
\end{aligned}
$$

where the relevant expansion parameter $\epsilon \ll 1$ is

$$
\epsilon=\frac{|q H|}{T^{2}}
$$

The quantities

$$
I_{\frac{3}{2}} \approx-0.610499, \quad b_{1} \approx 0.00581159, \quad I_{\frac{1}{2}} \approx-1.516256
$$

are defined in Eqs. (B23), (B24), and (B8), respectively.
The series is dominated by a term involving the half-integer power $\left(|q H| / T^{2}\right)^{3 / 2}$, a logarithmic term $|q H|^{2} / T^{4} \log |q H| / T^{2}$, and two terms quadratic in the magnetic field. If no magnetic field is present, the series reduces to the well-known pion gas contribution

$$
P(H=0)=\frac{\pi^{2}}{30} T^{4}+\mathcal{O}\left(T^{8} \log T\right) .
$$

As we illustrate by Fig. 4 in Appendix B, the representation (4.1) of the pressure - that includes the first three terms in the expansion of $\tilde{g}_{0}$ and $\tilde{g}_{1}$-provides a very accurate approximation not only for small values of $\epsilon$ (see Table II in Appendix B), but also for larger values of $\epsilon$ : $1 \%$ precision up to $\epsilon \approx 10$ is guaranteed, while $10 \%$ precision is still achieved up to $\epsilon \approx 20$.

\section{CONCLUSIONS}

Within chiral perturbation theory - based on a coordinate space representation for the thermal propagators-we have analyzed the impact of the magnetic field on the partition function up to the two-loop level. Using the dressed pion masses at zero temperature, we have shown that the pionpion interaction in the pressure may be attractive, repulsive, or zero. The respective sign of the two-loop interaction contribution is controlled by the strength of the magnetic field, as well as temperature and pion mass. In the weak magnetic field limit, the interaction is purely attractive at two-loop order, and gradually becomes weaker as the strength of the magnetic field increases.

We then have provided the expansion of the pressure in weak magnetic fields in the chiral limit. The dominant terms in the series are proportional to $\left(|q H| / T^{2}\right)^{3 / 2}$, $|q H|^{2} / T^{4} \log |q H| / T^{2}$, and $|q H|^{2} / T^{4}$.

The question arises whether three-loop corrections in the thermodynamic quantities-i.e., order- $p^{8}$ effects-are large compared to the two-loop results discussed here. While the corresponding three-loop analysis referring to zero magnetic field has been provided in Refs. [68,70], a three-loop analysis for QCD in the presence of a magnetic field, based on chiral perturbation theory, has never been attempted to the best of our knowledge. Work in this direction, relying on the coordinate space representation, is currently in progress.

\section{ACKNOWLEDGMENTS}

The author thanks G. S. Bali, J. Bijnens, T. Brauner, and H. Leutwyler for correspondence, as well as R. A. Sáenz and C. Castaño Bernard for illuminating discussions.

\section{APPENDIX A: FREE ENERGY DENSITY AT TWO LOOPS}

In this Appendix, we derive the order- $p^{6}$ contribution to the free energy density, originating from diagrams $6 A-6 C$ of Fig. 1. The two-loop diagram yields 


$$
z_{6 A}=\frac{M^{2}}{2 F^{2}} G_{1}^{ \pm} G_{1}^{0}-\frac{M^{2}}{8 F^{2}} G_{1}^{0} G_{1}^{0}
$$

where the thermal propagators $G_{1}^{ \pm}$for the charged pions and $G_{1}^{0}$ for the neutral pion are defined in Eq. (2.8). The result for the one-loop graph $6 B$,

$z_{6 B}=\left(4 l_{5}-2 l_{6}\right) \frac{|q H|^{2}}{F^{2}} G_{1}^{ \pm}+2 l_{3} \frac{M^{4}}{F^{2}} G_{1}^{ \pm}+l_{3} \frac{M^{4}}{F^{2}} G_{1}^{0}$,

involves various NLO effective constants $l_{i}$ that require renormalization (see below). The explicit structure of the tree-level contribution $z_{6 C}$ is not required here, because we are interested in the properties of the system at finite temperature.

In the decomposition of thermal propagators,

$$
\begin{aligned}
G_{1}^{ \pm} & =\Delta^{ \pm}(0)+\tilde{g}_{1}(M, T, H)+g_{1}(M, T, 0), \\
G_{1}^{0} & =\Delta^{0}(0)+g_{1}(M, T, 0),
\end{aligned}
$$

the kinematical functions are finite in the limit $d \rightarrow 4$. The zero-temperature propagators $\Delta^{ \pm}(0)$ and $\Delta^{0}(0)$, however, become singular and take the form

$$
\Delta^{ \pm}(0)=2 M^{2} \lambda+K_{1}, \quad \Delta^{0}(0)=2 M^{2} \lambda
$$

The integral $K_{1}$ and the parameter $\lambda$ are

$$
\begin{aligned}
K_{1}(M, H) & =\frac{|q H|^{\frac{d}{2}-1}}{(4 \pi)^{\frac{d}{2}}} \int_{0}^{\infty} \mathrm{d} \rho \rho^{-\frac{d}{2}+1} \exp \left(-\frac{M^{2}}{|q H|} \rho\right)\left(\frac{1}{\sinh (\rho)}-\frac{1}{\rho}\right) \\
\lambda & =\frac{1}{2}(4 \pi)^{-\frac{d}{2}} \Gamma\left(1-\frac{1}{2} d\right) M^{d-4} \\
& =\frac{M^{d-4}}{16 \pi^{2}}\left[\frac{1}{d-4}-\frac{1}{2}\left\{\ln 4 \pi+\Gamma^{\prime}(1)+1\right\}+\mathcal{O}(d-4)\right]
\end{aligned}
$$

Gathering results, the unrenormalized free energy density at order $p^{6}$ amounts to

$$
\begin{aligned}
z^{[6]}= & z_{6 A}+z_{6 B}+z_{6 C} \\
= & \frac{3 M^{2}}{8 F^{2}}\left(g_{1}\right)^{2}+\frac{M^{2}}{2 F^{2}} g_{1} \tilde{g}_{1}+g_{1}\left[\frac{3 M^{4}}{2 F^{2}} \lambda+\frac{M^{2}}{2 F^{2}} K_{1}+\left(4 l_{5}-2 l_{6}\right) \frac{|q H|^{2}}{F^{2}}+3 l_{3} \frac{M^{4}}{F^{2}}\right] \\
& +\tilde{g}_{1}\left[\frac{M^{4}}{F^{2}} \lambda+\left(4 l_{5}-2 l_{6}\right) \frac{|q H|^{2}}{F^{2}}+2 l_{3} \frac{M^{4}}{F^{2}}\right]+\frac{3 M^{6}}{2 F^{2}} \lambda^{2}+\frac{M^{4}}{F^{2}} K_{1} \lambda+\left(8 l_{5}-4 l_{6}\right) \frac{|q H|^{2} M^{2}}{F^{2}} \lambda+\left(4 l_{5}-2 l_{6}\right) \frac{|q H|^{2}}{F^{2}} K_{1} \\
& +6 l_{3} \frac{M^{6}}{F^{2}} \lambda+2 l_{3} \frac{M^{4}}{F^{2}} K_{1}+z_{6 C} .
\end{aligned}
$$

The first two terms are quadratic in the kinematical functions and are finite as $d$ approaches the physical dimension $d=4$. Considering the terms linear in $g_{1}$ and $\tilde{g}_{1}$,

$$
\begin{aligned}
& g_{1}\left[\frac{3 M^{4}}{2 F^{2}} \lambda+\frac{M^{2}}{2 F^{2}} K_{1}+\left(4 l_{5}-2 l_{6}\right) \frac{|q H|^{2}}{F^{2}}+3 l_{3} \frac{M^{4}}{F^{2}}\right], \\
& \tilde{g}_{1}\left[\frac{M^{4}}{F^{2}} \lambda+\left(4 l_{5}-2 l_{6}\right) \frac{|q H|^{2}}{F^{2}}+2 l_{3} \frac{M^{4}}{F^{2}}\right],
\end{aligned}
$$

using the standard convention for the renormalized NLO effective constants $\bar{l}_{i}$,

$$
l_{i}=\gamma_{i}\left(\lambda+\frac{\bar{l}_{i}}{32 \pi^{2}}\right), \quad \gamma_{3}=-\frac{1}{2}, \quad \gamma_{5}=-\frac{1}{6}, \quad \gamma_{6}=-\frac{1}{3},
$$

we arrive at

$$
+g_{1}\left[-\frac{3 \bar{l}_{3}}{64 \pi^{2}} \frac{M^{4}}{F^{2}}+\frac{M^{2}}{2 F^{2}} K_{1}+\frac{\bar{l}_{6}-\bar{l}_{5}}{48 \pi^{2}} \frac{|q H|^{2}}{F^{2}}\right]+\tilde{g}_{1}\left[-\frac{\bar{l}_{3}}{32 \pi^{2}} \frac{M^{4}}{F^{2}}+\frac{\bar{l}_{6}-\bar{l}_{5}}{48 \pi^{2}} \frac{|q H|^{2}}{F^{2}}\right] .
$$


Notice that the above expressions are perfectly finite: all divergences in Eq. (A7) have been canceled. Finally, the zero-temperature divergences contained in $z_{6 A}+z_{6 B}-$ displayed in the last two lines of Eq. (A6) - will be canceled by counterterms from the next-to-next-toleading-order Lagrangian $\mathcal{L}_{\text {eff }}^{6}$ contained in the zerotemperature contribution $z_{6 C}$.

\section{APPENDIX B: KINEMATICAL FUNCTIONS IN WEAK MAGNETIC FIELDS}

In this Appendix, we provide the representations for the kinematical functions in weak magnetic fields. From the very beginning, we operate in the chiral limit. The relevant functions in the free energy density are

$$
g_{0}(0, T, 0), \quad g_{1}(0, T, 0)
$$

that do not involve the magnetic field, and

$$
\tilde{g}_{0}(0, T, H), \quad \tilde{g}_{1}(0, T, H)
$$

that do depend on the magnetic field. The analysis for the former functions in the chiral limit has been given a long time ago in Ref. [68]:

$$
g_{0}(0, T, 0)=\frac{\pi^{2}}{45} T^{4}, \quad g_{1}(0, T, 0)=\frac{1}{12} T^{2} .
$$

The latter two functions are defined as

$$
\tilde{g}_{r}(0, T, H)=\frac{|q H|^{\frac{d}{2}-r}}{(4 \pi)^{\frac{d}{2}}} \int_{0}^{\infty} \mathrm{d} \rho \rho^{r-\frac{d}{2}}\left(\frac{1}{\sinh (\rho)}-\frac{1}{\rho}\right)\left[S\left(\frac{|q H|}{4 \pi T^{2} \rho}\right)-1\right],
$$

with

$$
S(z)=\sum_{n=-\infty}^{\infty} \exp \left(-\pi n^{2} z\right)
$$

The evaluation of $\tilde{g}_{1}(0, T, H)$ in weak magnetic fields has been established in Ref. [62] with the result

$$
\tilde{g}_{1}(0, T, H)=-\left\{\frac{\left|I_{\frac{1}{2}}\right|}{8 \pi^{3 / 2}} \sqrt{\epsilon}-\frac{\log 2}{16 \pi^{2}} \epsilon+\frac{\zeta(3)}{384 \pi^{4}} \epsilon^{2}-\frac{7 \zeta(7)}{98304 \pi^{8}} \epsilon^{4}+\mathcal{O}\left(\epsilon^{6}\right)\right\} T^{2} .
$$

The expansion parameter $\epsilon$ measures the ratio between magnetic field strength and temperature,

$$
\epsilon=\frac{|q H|}{T^{2}}
$$

By definition, in the weak magnetic field limit $|q H| \ll T^{2}$, this parameter is small. The integral $I_{\frac{1}{2}}$ is

$$
I_{\frac{1}{2}}=\int_{0}^{\infty} \mathrm{d} \rho \rho^{-1 / 2}\left(\frac{1}{\sinh (\rho)}-\frac{1}{\rho}\right) \approx-1.516256
$$

What remains to be done is the analogous expansion for $\tilde{g}_{0}(0, T, H)$. According to Ref. [62], the representation (B4) can be cast into the form

$$
\tilde{g}_{r}(0, T, H)=\frac{\epsilon}{(4 \pi)^{r+1}} T^{d-2 r} \int_{0}^{1} \mathrm{~d} \rho \rho^{-\frac{d}{2}+r}\left(\frac{1}{\sinh (\epsilon \rho / 4 \pi)}-\frac{4 \pi}{\epsilon \rho}\right)\left[S\left(\frac{1}{\rho}\right)-1\right]+\frac{\epsilon}{(4 \pi)^{r+1}} T^{d-2 r}\left\{I_{A}+I_{B}+I_{C}\right\},
$$

where the respective integrals are defined as

$$
\begin{aligned}
& I_{A}=\int_{0}^{1} \mathrm{~d} \rho \rho^{\frac{d}{2}-r-\frac{5}{2}}\left(\frac{1}{\sinh (\epsilon / 4 \pi \rho)}-\frac{4 \pi \rho}{\epsilon}\right)\left[S\left(\frac{1}{\rho}\right)-1\right], \\
& I_{B}=\int_{0}^{1} \mathrm{~d} \rho \rho^{\frac{d}{2}-r-\frac{5}{2}}\left(\frac{1}{\sinh (\epsilon / 4 \pi \rho)}-\frac{4 \pi \rho}{\epsilon}\right) \\
& I_{C}=-\int_{0}^{1} \mathrm{~d} \rho \rho^{\frac{d}{2}-r-2}\left(\frac{1}{\sinh (\epsilon / 4 \pi \rho)}-\frac{4 \pi \rho}{\epsilon}\right) .
\end{aligned}
$$


For $r=0$ and $d \rightarrow 4$, the first integral in Eq. (B9), much like the integral $I_{A}$, is well-defined. Following Ref. [62], the integral $I_{B}$ is split up into two terms:

$$
\begin{aligned}
I_{B} & =I_{B 1}+I_{B 2}, \\
I_{B 1} & =\frac{\epsilon^{\frac{d}{2}-r-\frac{3}{2}}}{(4 \pi)^{\frac{d}{2}-r-\frac{3}{2}}} \int_{0}^{\infty} \mathrm{d} \rho \rho^{-\frac{d}{2}+r+\frac{1}{2}}\left(\frac{1}{\sinh (\rho)}-\frac{1}{\rho}\right), \\
I_{B 2} & =-\int_{0}^{1} \mathrm{~d} \rho \rho^{-\frac{d}{2}+r+\frac{1}{2}}\left(\frac{1}{\sinh (\epsilon \rho / 4 \pi)}-\frac{4 \pi}{\epsilon \rho}\right) .
\end{aligned}
$$

For $r=0$ and $d \rightarrow 4$, we obtain

$$
\begin{aligned}
& I_{B 1}=\frac{\sqrt{\epsilon}}{\sqrt{4 \pi}} \int_{0}^{\infty} \mathrm{d} \rho \rho^{-\frac{3}{2}}\left(\frac{1}{\sinh (\rho)}-\frac{1}{\rho}\right), \\
& I_{B 2}=-\int_{0}^{1} \mathrm{~d} \rho \rho^{-\frac{3}{2}}\left(\frac{1}{\sinh (\epsilon \rho / 4 \pi)}-\frac{4 \pi}{\epsilon \rho}\right) .
\end{aligned}
$$

Note that the power $\sqrt{\epsilon}$ in $I_{B 1}$ is explicit, whereas $\epsilon$ appears in the integrand of $I_{B 2}$, as well as in the first integrand in Eq. (B9) and in $I_{A}$ of Eq. (B10), as an argument of the hyperbolic sine function. We thus Taylor-expand these pieces into

$$
\begin{aligned}
\frac{1}{\sinh (\epsilon \rho / 4 \pi)}-\frac{4 \pi}{\epsilon \rho}= & c_{1} \rho \epsilon+c_{2} \rho^{3} \epsilon^{3}+c_{3} \rho^{5} \epsilon^{5}+\mathcal{O}\left(\epsilon^{7}\right), \\
\frac{1}{\sinh (\epsilon / 4 \pi \rho)}-\frac{4 \pi \rho}{\epsilon}= & c_{1} \rho^{-1} \epsilon+c_{2} \rho^{-3} \epsilon^{3}+c_{3} \rho^{-5} \epsilon^{5} \\
& +\mathcal{O}\left(\epsilon^{7}\right)
\end{aligned}
$$

such that $\epsilon$-powers in all these integrals become explicit. The first few coefficients $c_{p}$ in the above series are

$$
\begin{aligned}
& c_{1}=-\frac{1}{24 \pi} \approx-1.33 \times 10^{-2}, \\
& c_{2}=\frac{7}{23040 \pi^{3}} \approx 9.80 \times 10^{-6}, \\
& c_{3}=-\frac{31}{15482880 \pi^{5}} \approx-6.54 \times 10^{-9}, \\
& c_{4}=\frac{127}{9909043200 \pi^{7}} \approx 4.24 \times 10^{-12}, \\
& c_{5}=-\frac{73}{896909967360 \pi^{9}} \approx-2.73 \times 10^{-15} .
\end{aligned}
$$

The last piece in the analysis of $\tilde{g}_{0}(0, T, H)$ in weak magnetic fields is $I_{C}$, defined in Eq. (B10). This integral for $r=0$, however, cannot be processed in the manner outlined in Ref. [62], which indeed worked for the case ${ }^{5} r=1$. Instead, we decompose the integral $I_{C}$

\footnotetext{
${ }^{5}$ In the decomposition $I_{C}=I_{C 1}+I_{C 2}$, Eq. (A15) of Ref. [62], both expressions $I_{C 1}$ and $I_{C 2}$ are singular if $r=0$ and $d \rightarrow 4$.
}

$$
I_{C}=-\int_{0}^{1} \mathrm{~d} \rho\left(\frac{1}{\sinh (\epsilon / 4 \pi \rho)}-\frac{4 \pi \rho}{\epsilon}\right)
$$

in an alternative way as

$$
\begin{aligned}
I_{C}(N)= & I_{C 1}(N)+I_{C 2}(N) \\
= & -\int_{0}^{N} \mathrm{~d} \rho\left(\frac{1}{\sinh (\epsilon / 4 \pi \rho)}-\frac{4 \pi \rho}{\epsilon}\right) \\
& +\int_{1}^{N} \mathrm{~d} \rho\left(\frac{1}{\sinh (\epsilon / 4 \pi \rho)}-\frac{4 \pi \rho}{\epsilon}\right),
\end{aligned}
$$

where $N \gg 1$. Redefining integration variables, we obtain

$$
\begin{aligned}
I_{C 1}(N)= & -\frac{\epsilon}{4 \pi} \int_{\epsilon / 4 \pi N}^{1} \mathrm{~d} \rho \rho^{-2}\left(\frac{1}{\sinh (\rho)}-\frac{1}{\rho}\right) \\
& -\frac{\epsilon}{4 \pi} \int_{1}^{\infty} \mathrm{d} \rho \rho^{-2}\left(\frac{1}{\sinh (\rho)}-\frac{1}{\rho}\right), \\
I_{C 2}(N)= & \frac{\epsilon}{4 \pi} \int_{\epsilon / 4 \pi N}^{\epsilon / 4 \pi} \mathrm{d} \rho \rho^{-2}\left(\frac{1}{\sinh (\rho)}-\frac{1}{\rho}\right) .
\end{aligned}
$$

The $N$ dependence cancels in the sum $I_{C 1}(N)+I_{C 2}(N)$, and we are left with

$$
\begin{aligned}
I_{C}= & \frac{\epsilon}{4 \pi} \int_{1}^{\epsilon / 4 \pi} \mathrm{d} \rho \rho^{-2}\left(\frac{1}{\sinh (\rho)}-\frac{1}{\rho}\right) \\
& -\frac{\epsilon}{4 \pi} \int_{1}^{\infty} \mathrm{d} \rho \rho^{-2}\left(\frac{1}{\sinh (\rho)}-\frac{1}{\rho}\right) .
\end{aligned}
$$

In the second contribution, the power $\epsilon$ is explicit. In the first contribution, where $\epsilon$ appears in the upper integration limit, we Taylor-expand the integrand, and then integrate term by term. The final result for $I_{C}$ can be cast into the form

$$
\begin{aligned}
I_{C}= & -\frac{\epsilon}{24 \pi} \log \left(\frac{\epsilon}{4 \pi}\right)+\frac{\hat{J}-\hat{I}}{4 \pi} \epsilon \\
& -\sum_{n=2}^{\infty} \frac{2^{2 n-1}-1}{(n-1)(2 n) !} \frac{B_{2 n}}{(4 \pi)^{2 n-1}} \epsilon^{2 n-1},
\end{aligned}
$$

where the $B_{2 n}$ 's are Bernoulli numbers and the quantities $\hat{J}$ and $\hat{I}$ are defined as

$$
\begin{aligned}
& \hat{J}=\sum_{n=2}^{\infty} \frac{2^{2 n-1}-1}{(n-1)(2 n) !} B_{2 n} \approx-0.00924219, \\
& \hat{I}=\int_{1}^{\infty} \mathrm{d} \rho \rho^{-2}\left(\frac{1}{\sinh (\rho)}-\frac{1}{\rho}\right) \approx-0.179499 .
\end{aligned}
$$

Note that the structure of the $\epsilon$-expansion of $I_{C}$ is now manifest.

Collecting individual contributions, after some algebra, and with the help of the identity 


$$
\frac{2}{\pi^{\frac{z}{2}}} \Gamma\left(\frac{z}{2}\right) \zeta(z)=\int_{0}^{\infty} \mathrm{d} \rho \rho^{\frac{z}{2}-1}[S(\rho)-1],
$$

the expansion of the kinematical function $\tilde{g}_{0}(0, T, H)$ in weak magnetic fields and in the chiral limit takes the form

$$
\begin{aligned}
\tilde{g}_{0}(0, T, H)= & \left\{-\frac{\left|I_{\frac{3}{2}}\right|}{8 \pi^{3 / 2}} \epsilon^{\frac{3}{2}}-\frac{1}{96 \pi^{2}} \epsilon^{2} \log \epsilon+b_{1} \epsilon^{2}\right. \\
& \left.+b_{2} \epsilon^{4}+b_{3} \epsilon^{6}+b_{4} \epsilon^{8}+\mathcal{O}\left(\epsilon^{10}\right)\right\} T^{4},
\end{aligned}
$$

where

$$
I_{\frac{3}{2}}=\int_{0}^{\infty} \mathrm{d} \rho \rho^{-\frac{3}{2}}\left(\frac{1}{\sinh (\rho)}-\frac{1}{\rho}\right) \approx-0.610499 .
$$

The coefficient $b_{1}$ is

$$
b_{1}=\frac{6(\hat{J}-\hat{I})-\tilde{I}+\log 4 \pi}{96 \pi^{2}} \approx 0.00581159,
$$

with

$$
\tilde{I}=\int_{0}^{1} \mathrm{~d} \rho\left(\rho^{-1}+\rho^{-\frac{3}{2}}\right)\left[S\left(\frac{1}{\rho}\right)-1\right]-\int_{0}^{1} \mathrm{~d} \rho \rho^{-\frac{1}{2}},
$$

while the coefficients $b_{p}(p \geq 2)$ are

$$
b_{p}=-\frac{2\left(2^{2 p-1}-1\right)}{(4 \pi)^{2 p}(2 p) !}\left\{\frac{2 \Gamma\left(2 p-\frac{3}{2}\right) \zeta(4 p-3)}{\pi^{2 p-\frac{3}{2}}}+\frac{1}{1-p}\right\} B_{2 p},
$$$$
p \geq 2 \text {. }
$$

The numerical values of the first five coefficients $b_{p}(p \geq 2)$ are given in Table I.

More explicitly, the series can be written as

$$
\begin{aligned}
\tilde{g}_{0}(0, T, H)= & \left\{-\frac{\left|I_{\frac{3}{2}}\right|}{8 \pi^{3 / 2}} \epsilon^{\frac{3}{2}}-\frac{1}{96 \pi^{2}} \epsilon^{2} \log \epsilon\right. \\
& +\frac{6(\hat{J}-\hat{I})-\tilde{I}+\log 4 \pi}{96 \pi^{2}} \epsilon^{2} \\
& -\frac{7\left(2 \pi^{2}-3 \zeta(5)\right)}{184320 \pi^{6}} \epsilon^{4}+\frac{31\left(4 \pi^{4}-105 \zeta(9)\right)}{495452160 \pi^{10}} \epsilon^{6} \\
& \left.-\frac{127\left(32 \pi^{6}-31185 \zeta(13)\right)}{3805072588800 \pi^{14}} \epsilon^{8}+\mathcal{O}\left(\epsilon^{10}\right)\right\} T^{4} .
\end{aligned}
$$

To check convergence properties of the above series for $\tilde{g}_{0}(0, T, H)$ in the weak magnetic field limit $|q H| \ll T^{2}$, let us compare the first few terms in the $\epsilon$-expansion with the exact result Eq. (B4). The first column in Table II displays
TABLE I. The first five coefficients $b_{p}$ defined by Eq. (B26).

\begin{tabular}{cc}
\hline \hline$p$ & $b_{p}$ \\
\hline 2 & $-6.56867042287 \times 10^{-7}$ \\
3 & $1.90033315207 \times 10^{-10}$ \\
4 & $1.55270844266 \times 10^{-15}$ \\
5 & $-3.08314759762 \times 10^{-16}$ \\
6 & $1.87712447343 \times 10^{-18}$ \\
\hline \hline
\end{tabular}

the exact result, while the second column just takes into account the leading term in the series (B27) proportional to $\epsilon^{3 / 2}$. The third column, furthermore, incorporates the $\epsilon^{2} \log \epsilon$ contribution, and the fourth column finally extends up to the $\epsilon^{2}$ term. One observes that a very good approximation is achieved by just including the first three terms: the series (B27) converges quite rapidly.

Finally, it should be noted that the order- $T^{4}$ contribution in the pressure-i.e., the coefficient $p_{1}$ in Eq. (3.4),

$$
\begin{aligned}
p_{1}\left(t, m, m_{H}\right)= & h_{0}\left(M_{\pi}^{ \pm}, T, 0\right)+\frac{1}{2} h_{0}\left(M_{\pi}^{0}, T, 0\right) \\
& +\tilde{h}_{0}\left(M_{\pi}^{ \pm}, T, H\right),
\end{aligned}
$$

contains the kinematical functions $h_{0}\left(M_{\pi}^{ \pm}, T, 0\right)$ and $\tilde{h}_{0}\left(M_{\pi}^{ \pm}, T, H\right)$, which, in the chiral limit, reduce to

$$
h_{0}\left(M_{H}, T, 0\right), \quad \tilde{h}_{0}\left(M_{H}, T, H\right),
$$

with

$$
\left(M_{H}\right)^{2}=\frac{\bar{l}_{6}-\bar{l}_{5}}{48 \pi^{2}} \frac{|q H|^{2}}{F^{2}} .
$$

In the weak magnetic field limit, the kinematical function $h_{0}\left(M_{H}, T, 0\right)$ hence takes the form

$$
\begin{aligned}
h_{0}\left(M_{H}, T, 0\right)= & h_{0}(0, T, 0)-\alpha \epsilon^{2} h_{1}(0, T, 0) \\
& +\frac{\alpha^{2} \epsilon^{4}}{2} h_{2}(0, T, 0)+\mathcal{O}\left(\epsilon^{6}\right),
\end{aligned}
$$

where

$$
\alpha=\frac{\bar{l}_{6}-\bar{l}_{5}}{12 \pi} t^{2}, \quad \epsilon=\frac{|q H|}{T^{2}}, \quad t=\frac{T}{4 \pi F} .
$$

Analogously, in the weak magnetic field limit, the kinematical function $\tilde{h}_{0}\left(M_{H}, T, H\right)$ amounts to

$$
\begin{aligned}
\tilde{h}_{0}\left(M_{H}, T, H\right)= & \tilde{h}_{0}(0, T, H)-\alpha \epsilon^{2} \tilde{h}_{1}(0, T, H) \\
& +\frac{\alpha^{2} \epsilon^{4}}{2} \tilde{h}_{2}(0, T, H)+\mathcal{O}\left(\epsilon^{6}\right) .
\end{aligned}
$$

We hence have additional terms in the weak magnetic field expansion of the pressure in the chiral limit, which contains the NLO low-energy constants $\bar{l}_{5}$ and $\bar{l}_{6}$. 
TABLE II. Exact result and leading terms in the series (B27) for the kinematical function $\tilde{g}_{0}$ in the limit $|q H| \ll T^{2}$. We use the notation where $-3.50963233726-4$ stands for $-3.50963233726 \times 10^{-4}$, etc.

\begin{tabular}{lccrr}
\hline \hline$\epsilon$ & $\tilde{g}_{0} / T^{4}$ & $\mathcal{O}\left(\epsilon^{3 / 2}\right)$ & $\mathcal{O}\left(\epsilon^{2} \log \epsilon\right)$ & $\mathcal{O}\left(\epsilon^{2}\right)$ \\
\hline 0.1 & $-3.50963233726-4$ & $-4.33381291264-4$ & $-4.09079140529-4$ & $-3.50963246014-4$ \\
0.05 & $-1.30789993783-4$ & $-1.53223424946-4$ & $-1.45318968180-4$ & $-1.30789994551-4$ \\
0.01 & $-1.26375177959-5$ & $-1.37047197570-5$ & $-1.32186767423-5$ & $-1.26375177971-5$ \\
0.005 & $-4.56026045635-6$ & $-4.84535013722-6$ & $-4.70555019271-6$ & $-4.56026045643-6$ \\
0.001 & $-4.20279056592-7$ & $-4.33381291264-7$ & $-4.26090646044-7$ & $-4.20279056592-7$ \\
0.0005 & $-1.49764974370-7$ & $-1.53223424946-7$ & $-1.51217871733-7$ & $-1.49764974370-7$ \\
0.0001 & $-1.35493952595-8$ & $-1.37047197570-8$ & $-1.36075111541-8$ & $-1.35493952595-8$ \\
\hline \hline
\end{tabular}

As Table II demonstrates, the first three terms in the series (B27) give a very good approximation for the function $\tilde{g}_{0}$ when the expansion parameter $\epsilon$ is small. In order to assess the quality of our expansion for larger values of $\epsilon$, we consider the quantity $\xi_{0}$, defined as the ratio between the sum of the first three terms in the expansion (B27) with respect to the full (unexpanded) function $\tilde{g}_{0}$. This ratio corresponds to the red curve shown in Fig. 4.
In the same figure, we also plot (in blue) the quantity $\xi_{1}$, which corresponds to the analogous ratio for the function $\tilde{g}_{1}$-i.e., the ratio between the sum of the first three terms in the expansion (B6) with respect to the full (unexpanded) function $\tilde{g}_{1}$. One observes that in either case, the first three terms in the series provide a very good approximation even for larger values of $\epsilon$. Precision of $1 \%$ is guaranteed up to $\epsilon \approx 10 ; 10 \%$ precision is still achieved up to $\epsilon \approx 20$.
[1] I. A. Shushpanov and A. V. Smilga, Phys. Lett. B 402, 351 (1997).

[2] N. O. Agasian, Phys. Lett. B 488, 39 (2000).

[3] N. O. Agasian, Phys. At. Nucl. 64, 554 (2001).

[4] N. O. Agasian and I. A. Shushpanov, J. High Energy Phys. 10 (2001) 006.

[5] T. D. Cohen, D. A. McGady, and E. S. Werbos, Phys. Rev. C 76, 055201 (2007).

[6] N. O. Agasian, Phys. At. Nucl. 71, 1967 (2008).

[7] N. O. Agasian and S. M. Fedorov, Phys. Lett. B 663, 445 (2008).

[8] N. O. Agasian, Phys. At. Nucl. 72, 339 (2009).

[9] J. O. Andersen, Phys. Rev. D 86, 025020 (2012).

[10] J. O. Andersen, J. High Energy Phys. 10 (2012) 005.

[11] T. Brauner and S. V. Kadam, J. High Energy Phys. 11 (2017) 103.

[12] M. A. Andreichikov and Y. A. Simonov, Eur. Phys. J. C 78, 902 (2018).

[13] M. D'Elia, S. Mukherjee, and F. Sanfilippo, Phys. Rev. D 82, 051501 (2010).

[14] M. D'Elia and F. Negro, Phys. Rev. D 83, 114028 (2011).

[15] G. S. Bali, F. Bruckmann, G. Endrödi, Z. Fodor, S. D. Katz, S. Krieg, A. Schäfer, and K. K. Szabó, J. High Energy Phys. 02 (2012) 044.

[16] V. V. Braguta, P. V. Buividovich, T. Kalaydzhyan, S. V. Kuznetsov, and M. I. Polikarpov, Phys. At. Nucl. 75, 488 (2012).

[17] G. S. Bali, F. Bruckmann, G. Endrödi, Z. Fodor, S. D. Katz, and A. Schäfer, Phys. Rev. D 86, 071502 (2012).
[18] G. S. Bali, F. Bruckmann, M. Constantinou, M. Costa, G. Endrödi, Z. Fodor, S. D. Katz, H. Panagopoulos, and A. Schäfer, Phys. Rev. D 86, 094512 (2012).

[19] G. S. Bali, F. Bruckmann, G. Endrödi, F. Gruber, and A. Schäfer, J. High Energy Phys. 04 (2013) 130.

[20] V. G. Bornyakov, P. V. Buividovich, N. Cundy, O. A. Kochetkov, and A. Schäfer, Phys. Rev. D 90, 034501 (2014).

[21] G. S. Bali, F. Bruckmann, G. Endrödi, S. D. Katz, and A. Schäfer, J. High Energy Phys. 08 (2014) 177.

[22] C. Bonati, M. D'Elia, M. Mariti, F. Negro, and F. Sanfilippo, Phys. Rev. D 89, 054506 (2014).

[23] E.-M. Ilgenfritz, M. Müller-Preussker, B. Petersson, and A. Schreiber, Phys. Rev. D 89, 054512 (2014).

[24] M. D'Elia, F. Manigrasso, F. Negro, and F. Sanfilippo, Phys. Rev. D 98, 054509 (2018).

[25] G. Endrödi, M. Giordano, S. D. Katz, T. C. Kovács, and F. Pittler, J. High Energy Phys. 07 (2019) 007.

[26] R. Gatto and M. Ruggieri, Phys. Rev. D 83, 034016 (2011).

[27] A. Amador and J. O. Andersen, Phys. Rev. D 88, 025016 (2013).

[28] M. Ferreira, P. Costa, D. P. Menezes, C. Providencia, and N. N. Scoccola, Phys. Rev. D 89, 016002 (2014).

[29] M. Ferreira, P. Costa, O. Lourenco, T. Frederico, and C. Providencia, Phys. Rev. D 89, 116011 (2014).

[30] M. Ferreira, P. Costa, and C. Providencia, Phys. Rev. D 90, 016012 (2014).

[31] E. J. Ferrer, V. de la Incera, I. Portillo, and M. Quiroz, Phys. Rev. D 89, 085034 (2014). 
[32] M. Ruggieri, Z. Y. Lu, and G. X. Peng, Phys. Rev. D 94, 116003 (2016).

[33] R. Zhang, W. Fu, and Y. Liu, Eur. Phys. J. C 76, 307 (2016).

[34] T. D. Cohen and E. S. Werbos, Phys. Rev. C 80, 015203 (2009).

[35] A. J. Mizher, M. N. Chernodub, and E. S. Fraga, Phys. Rev. D 82, 105016 (2010).

[36] S. Nam and C.-W. Kao, Phys. Rev. D 87, 114003 (2013).

[37] M. Frasca and M. Ruggieri, Phys. Rev. D 83, 094024 (2011).

[38] E. S. Fraga and L. F. Palhares, Phys. Rev. D 86, 016008 (2012).

[39] F. Bruckmann, G. Endrödi, and T. G. Kovács, J. High Energy Phys. 04 (2013) 112.

[40] G. Endrödi, J. High Energy Phys. 04 (2013) 023.

[41] V. D. Orlovsky and Y. A. Simonov, J. High Energy Phys. 09 (2013) 136.

[42] J.-P. Blaizot, E. S. Fraga, and L. F. Palhares, Phys. Lett. B 722, 167 (2013).

[43] M. Ruggieri, M. Tachibana, and V. Greco, J. High Energy Phys. 07 (2013) 165.

[44] G. S. Bali, F. Bruckmann, G. Endrödi, and A. Schäfer, Phys. Rev. Lett. 112, 042301 (2014).

[45] G. Colucci, E. S. Fraga, and A. Sedrakian, Phys. Lett. B 728, 19 (2014).

[46] V. D. Orlovsky and Y. A. Simonov, Phys. Rev. D 89, 074034 (2014).

[47] V. D. Orlovsky and Y. A. Simonov, Phys. Rev. D 89, 054012 (2014).

[48] A. Haber, F. Preis, and A. Schmitt, Phys. Rev. D 90, 125036 (2014).

[49] N. Mueller and J. M. Pawlowski, Phys. Rev. D 91, 116010 (2015).

[50] V. D. Orlovsky and Y. A. Simonov, Int. J. Mod. Phys. A 30, 1550060 (2015).
[51] Y. A. Simonov and V. D. Orlovsky, JETP Lett. 101, 423 (2015).

[52] K. Kamikado and T. Kanazawa, J. High Energy Phys. 01 (2015) 129.

[53] L.-G. Pang, G. Endrödi, and H. Petersen, Phys. Rev. C 93, 044919 (2016).

[54] A. N. Tawfik, A. M. Diab, N. Ezzelarab, and A. G. Shalaby, Adv. High Energy Phys. 2016, 1381479 (2016).

[55] A. Bandyopadhyay, N. Haque, and M. G. Mustafa, Phys. Rev. D 100, 034031 (2019).

[56] S. Rath and B. K. Patra, J. High Energy Phys. 12 (2017) 098.

[57] M. A. Andreichikov and Y. A. Simonov, Eur. Phys. J. C 78, 420 (2018).

[58] F. L. Braghin, Eur. Phys. J. A 54, 45 (2018).

[59] S. Rath and B. K. Patra, Eur. Phys. J. A 55, 220 (2019).

[60] B. Karmakar, R. Ghosh, A. Bandyopadhyay, N. Haque, and M. G. Mustafa, Phys. Rev. D 99, 094002 (2019).

[61] J. O. Andersen, W. R. Naylor, and A. Tranberg, Rev. Mod. Phys. 88, 025001 (2016).

[62] C. P. Hofmann, Phys. Rev. D 99, 014030 (2019).

[63] J. Bijnens (private communication); R. Andersson, Lund University bachelor thesis (to be published).

[64] H. Leutwyler, in Hadron Physics 94: Topics on the Structure and Interaction of Hadronic Systems, edited by V. E. Herscovitz, C. A. Z. Vasconcellos, and E. Ferreira (World Scientific, Singapore, 1995), p. 1.

[65] S. Scherer, Adv. Nucl. Phys. 27, 277 (2003).

[66] J. Gasser and H. Leutwyler, Ann. Phys. (N.Y.) 158, 142 (1984).

[67] J. Bijnens, G. Colangelo, and G. Ecker, Ann. Phys. (N.Y.) 280, 100 (2000).

[68] P. Gerber and H. Leutwyler, Nucl. Phys. B321, 387 (1989).

[69] S. Aoki et al., Eur. Phys. J. C 80, 113 (2020).

[70] C. P. Hofmann, Nucl. Phys. B916, 254 (2017). 